\title{
Continuum emission from irradiated solid deuterium
}

\author{
J. A. Forrest, R. L. Brooks, and J. L. Hunt \\ Department of Physics, University of Guelph, Guelph, Ontario, Canada N1G 2WI \\ B. Stenum, J. Schou, H. Sørensen, and P. Gürtler* \\ Physics Department, EURATOM-Risø National Laboratory, DK-4000, Roskilde, Denmark \\ F. Magnotta, E. R. Mapoles, P. C. Souers, and G. W. Collins \\ Lawrence Livermore National Laboratory, University of California, Livermore, California 94550
}

(Received 17 July 1992)

\begin{abstract}
A new emission feature from the spectrum of irradiated solid deuterium has been observed in the very near-infrared spectral region. Experiments from three laboratories, using different excitation conditions, have confirmed the observation. Comparison of the timing and temperature dependence of the spectral feature to the information previously available from electron spin resonance studies of solid deuterium, points to atomic association as the underlying cause. We shall show the connection of this emission to the occurrence of thermal spikes and optical flashes, previously observed in solid deuterium.
\end{abstract}

\section{INTRODUCTION}

Spectroscopy of solid hydrogens irradiated with highenergy radiation has revealed the presence of many spectral features not found in the unirradiated solid. Radiation-induced lines in the fundamental absorption spectrum of solid deuterium led to the discovery of immobile charge species in the solid. ${ }^{1,2}$ Further experimental investigations in the infrared and ultraviolet spectral regions, ${ }^{3-7}$ as well as theoretical studies, ${ }^{8-10}$ have strengthened this interpretation. Studies of these features after the irradiation is terminated show that the charge species last for many minutes or even hours. A model derived previously ${ }^{4}$ explaining the formation of these charges is able to give satisfactory agreement with the observed results. Whereas that model did not explicitly consider atom concentration as a variable of interest, nothing precludes the model from doing so. The model would allow for a substantial atom concentration but, at the time, no experimental signature of atoms was available.

Unpaired hydrogen atoms in a solid hydrogen sample were observed by Jen et al. ${ }^{11}$ in the ESR spectrum of condensed microwave discharge products. Since then atoms have also been observed in solids irradiated by electrons $^{12}$ and gamma radiation. ${ }^{13}$ The first evidence that solid hydrogen matrices could store large numbers of atoms came when Webeler observed rapid energy releases in tritiated solid hydrogen at temperatures between 0.2 and $0.8 \mathrm{~K}^{14}$ Webeler interpreted these energy releases, as much as $0.3 \mathrm{~J}$ for a $1-\mathrm{cm}^{3}$ sample, as originating from the recombination of atoms in the solid. Theoretical work based on Webeler's results ${ }^{15-17}$ predicts atom concentrations between $10^{16}$ and $10^{18} \mathrm{~cm}^{-3}$. More recently, Collins et al. ${ }^{18}$ have observed triggered thermal energy releases in solid $\mathrm{D} / \mathrm{T}$ mixtures at temperatures between 1.2 and $5 \mathrm{~K}$. These thermal spikes were found to be correlated to decreases in the atom concentration corresponding to the recombination of about 300 ppm (ap- proximately $3 \times 10^{18} \mathrm{~cm}^{-3}$ ) of stored atoms. These samples have also shown triggered optical pulses in which bright flashes of light are emitted from the sample following a triggering thermal pulse. ${ }^{19}$ The optical flashes consist of a broad emission feature centered at $\sim 1.48 \mathrm{eV}$ with FWHM of $0.25 \mathrm{eV}$. No other flash emission is observed over the spectral range of $250-1700 \mathrm{~nm}$. These optical flashes are believed to be related to the thermal pulses, and as such related to the $\mathrm{D}$ atom concentration. This work reports the spectrum of these optical flashes and a detailed study of a steady-state emission associated with these flashes.

\section{EXPERIMENT}

Experimental studies on the red emission were performed independently at three different laboratories, each employing a different method of irradiation. Experiments at the Lawrence Livermore National Laboratory use tritium doping, those at Ris $\varnothing$ use electron-beam irradiation, and those performed by the University of Guelph researchers use proton-beam irradiation. Details regarding these experiments have been published previously. $4,7,18,19$ Brief descriptions, incorporating changes for these experiments, are included here.

The experimental setup at Ris $\varnothing$ is shown in Fig. 1. The electron beam is deflected through a $15^{\circ}$ angle so that light emitted from the filament is not detected by the monochromator. Electron-beam energies of $1-3 \mathrm{keV}$ at currents of $50-300 \mathrm{nA}$ struck a thick film $(\sim 1 \mu \mathrm{m})$ of solid deuterium deposited on a gold substrate suspended below a pumped liquid-helium cryostat. Optical detection was by a $0.3-\mathrm{m} \mathrm{McPherson}$ model 218 monochromator with a $1200-1 / \mathrm{mm}$ holographic grating and a Hamamatsu R943-02 photomultiplier and standard photon counting.

For the Guelph experiment, protons from the McMaster Tandem Accelerator passed through a thin $\mathrm{Ni}$ foil and entered a $1-\mathrm{cm}^{3}$ sample of solid $\mathrm{D}_{2}$ with an energy of 


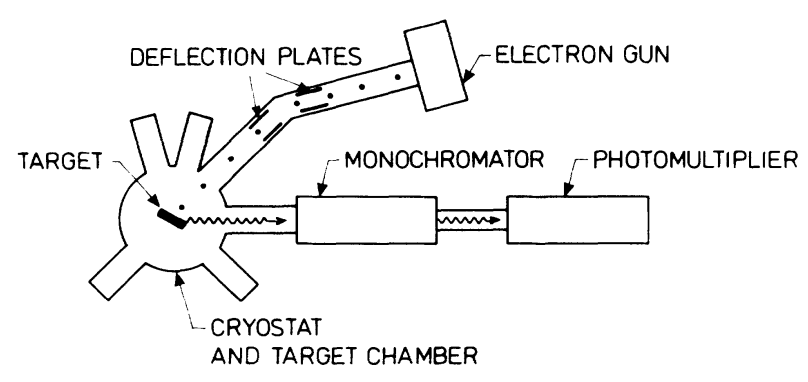

FIG. 1. Experimental apparatus at Ris $\varnothing$ for measuring emission from solid deuterium.

about $15 \mathrm{MeV}$ at a current of 6-10 nA. Radiation emitted from the sample at right angles to the proton beam was focused onto the entrance slit of a similar McPherson monochromator equipped with a $600-1 / \mathrm{mm}$ grating blazed at $1.0 \mu \mathrm{m}$. The dispersed light at the exit slit was focused onto the photocathode of an RCA C31034 photomultiplier. The signal from the photomultiplier is processed in one of two ways: For photon counting the output is first preamplified then input to a Stanford Instruments SR-400 photon counter. For some timing studies the output from the PMT is shunted through a $15-\mathrm{K} \Omega$ resistor, and the resulting voltage signal is input to a Stanford Instruments SR-510 lock-in amplifier.

In order to study the behavior of the red emission upon initiation and termination of the irradiation, two types of timing measurements are made at Guelph. To examine long-time behavior the monochromator is set to the wavelength of maximum emission, the beam is turned on (or off), and the signal from the photon counter is monitored as a function of time. By this type of timing measurement, times from about $0.1 \mathrm{sec}$ to many thousands of seconds can be recorded. For short-time behavior the monochromator is again set to the wavelength of the maximum emission and the proton beam is pulsed by an electrostatic chopper at frequencies ranging from $\mathrm{kHz}$ to $0.1 \mathrm{~Hz}$. The resulting ac signal is amplified, but not filtered, by the lock-in amplifier, and input to a transient digitizer with averaging capabilities. This system is capable of measuring time scales from $10^{-7}$ to $10^{-2} \mathrm{sec}$.

At LLNL, in order to study the red emission before, during, and after an optical flash, tritiated samples were monitored by both charge-coupled device (CCD) and Germanium arrays and spectra recorded at constant time intervals. These experiments also involved simultaneous recording of the temperature and optical signal in order to determine a relationship between these optical flashes and the previously mentioned thermal spikes.

\section{RESULTS}

The spectrum of the steady-state red emission in irradiated solid deuterium, as obtained in all three laboratories, is shown in Fig. 2. This broad continuum spectrum has not been observed in gaseous deuterium nor does it correspond to any previously observed spectrum in deuterium. Air impurities can cause observable spectral features in irradiated solid deuterium ${ }^{20}$ but the air concentration was

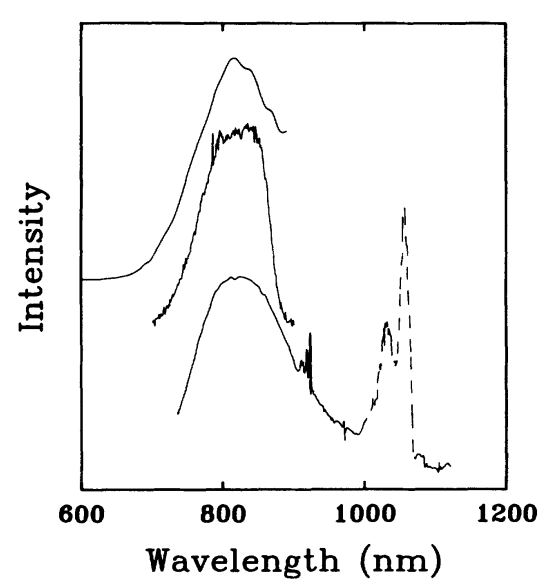

FIG. 2. Steady-state red emission. The top spectrum, obtained from Ris $\emptyset$, has been calibrated for system response. The proton-irradiated sample is in the middle, and the tritiated sample is on the bottom. The latter has been corrected for detector and monochromator response. The line emission features originate from known $\mathrm{He}_{2}$ transitions.

below the threshold of detectibility for the known lines. Furthermore, the intensity of this spectrum did not increase when samples were doped with nitrogen or oxygen impurities at Ris $\varnothing .{ }^{21}$ Uncalibrated spectra from all three laboratories are similar in both shape and intensity.

CCD array spectra of the red emission, using tritiumdoped samples at LLNL, taken at three time intervals during a triggered flash are shown in Fig. 3 and demonstrate that the optical flashes mentioned earlier have essentially the same spectrum as the steady-state red emission. It can also be seen from this series of spectra that after a flash occurs the steady-state emission temporarily disappears. Within the time resolution of these experiments the optical flashes are observed to occur simultaneously with thermal spikes. However, at $2.4 \mathrm{~K}$, spontaneous heat spikes occur sometimes, without change in the steady-state component of the red emission.

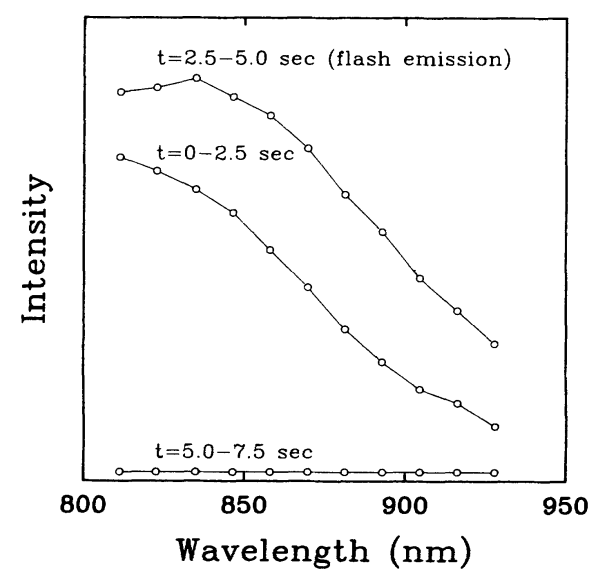

FIG. 3. Red emission during an optical flash using a tritiated sample, integrated over $2.5 \mathrm{sec}$ per frame. 
Previous studies on the optical pulses indicate that the steady-state emission has an intensity between 1 and 3 orders of magnitude smaller than that of the flashes, depending on the initial temperature. The integrated pulse energy remains constant with temperature over the measured range 2.4-4.2 $\mathrm{K}$. The amount of energy in the $800-\mathrm{nm}$ optical pulse is $\sim 0.2 \%$ of the available recombination energy. ${ }^{19}$ No steady-state emission in the spectral range studied has been observed in solid hydrogen (protium) samples to date at temperatures as low as 4.2 $\mathrm{K}$, and studies of optical flashes have not been carried out in hydrogen (protium) samples. The mechanism for this broad emission spectrum in deuterium remains uncertain but the ultimate cause appears to be association of two D atoms in the solid.

Uncalibrated emission spectra, using proton-beam irradiation, as a function of sample cell temperature, are shown in Fig. 4. The actual sample temperatures may be slightly higher than these values due to the thermal conductivity of the sample and heating by radiation, but are estimated to be within about $0.5 \mathrm{~K}$ of these values. Similar spectra have been observed at LLNL using tritium doping.

Figure 5 shows the turn on of the red emission on an annealed (12 $\mathrm{K}$ for $10 \mathrm{~min}$ ) sample using proton-beam irradiation. This shows that the red emission grows in much faster than any of the charge features previously observed. The turn on of the red emission on a previously unirradiated sample is also shown in Fig. 5. This turn on is different from that of the annealed sample and suggests that annealing the sample, while it does eliminate the charged species, ${ }^{2-4,6}$ does not get rid of some fraction of the species responsible for the red emission. The time scale for radiation-induced damage is typically of the order of minutes to hours, and since both the annealed and fresh sample show a saturated emission after only about $50 \mathrm{sec}$, the difference between them is not believed to be related to crystal damage alone. This same behavior has been seen to exist for $\mathrm{N}$ and $\mathrm{O}$ impurities in solid hydro-

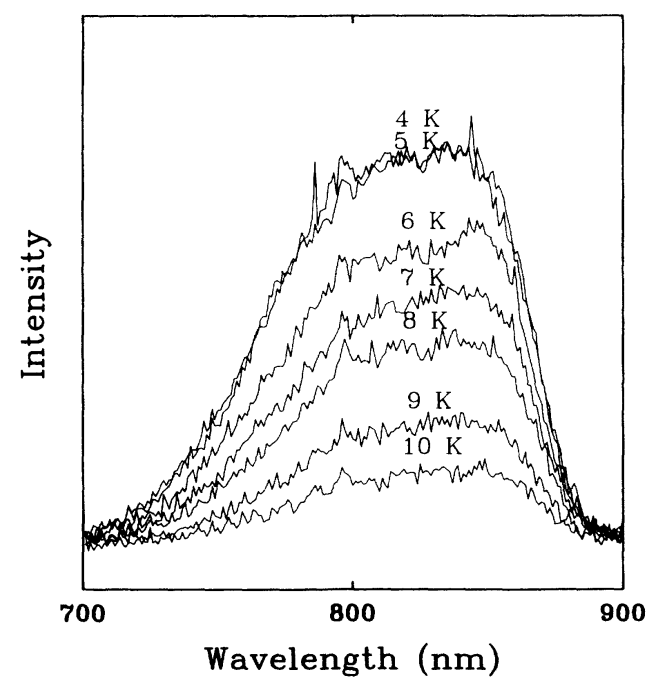

FIG. 4. Red emission spectra as a function of sample cell temperature taken with proton-beam irradiation.

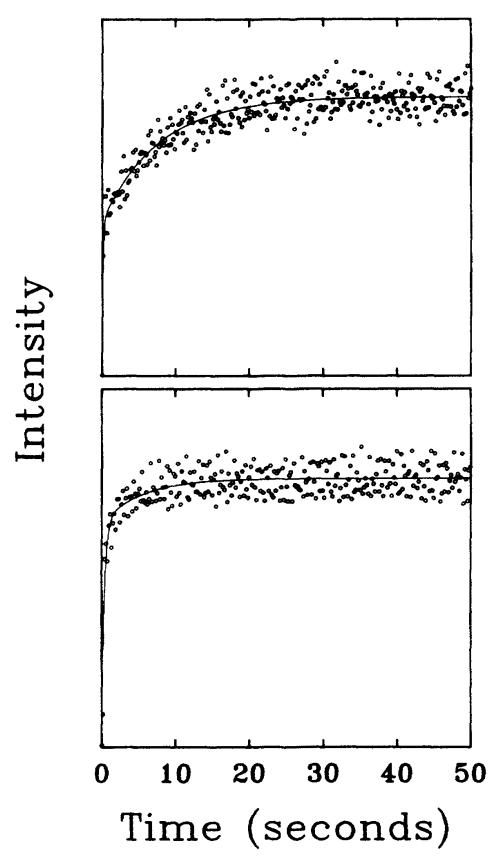

FIG. 5. Slow timing behavior of red emission spectra, taken with proton-beam irradiation. The top curve is for a previously unirradiated sample, and the bottom curve is for an annealed sample. The temperature of the sample is $4 \mathrm{~K}$.

gen hosts ${ }^{20}$ where the sample had to be melted to eliminate the atomic species. A multiexponential fit to the initial turn on of the fresh sample shows two components: a fast component growing in too quickly to measure (lifetime $<0.15 \mathrm{sec}$ ), as well as a slower (lifetime $=7 \mathrm{sec}$ ) component. The fast growth component cannot be accurately measured with this timing technique. In the LLNL experiments, the steady-state emission returns to full intensity in 8-10 min following a flash for $50 / 50$ samples of $\mathrm{D}_{2} / \mathrm{T}_{2}$ (see Fig. 3). The intensity buildup rate following a flash is related to the tritium concentration in the sample.

Fast timing measurements on a previously proton-

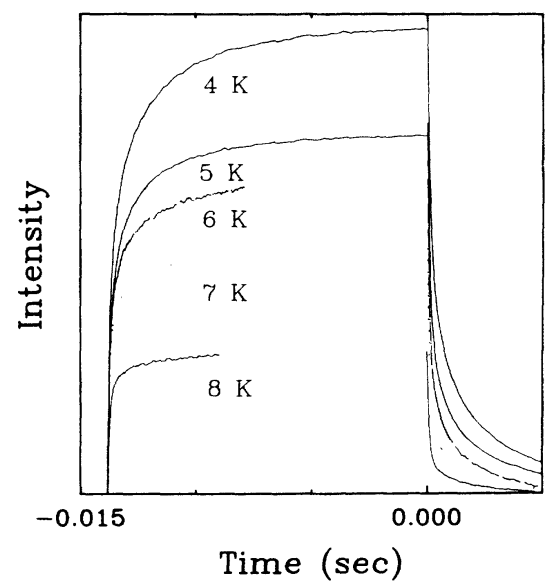

FIG. 6. Fast timing curves at various temperatures. Protonbeam irradiation stopped at $t=0$. 


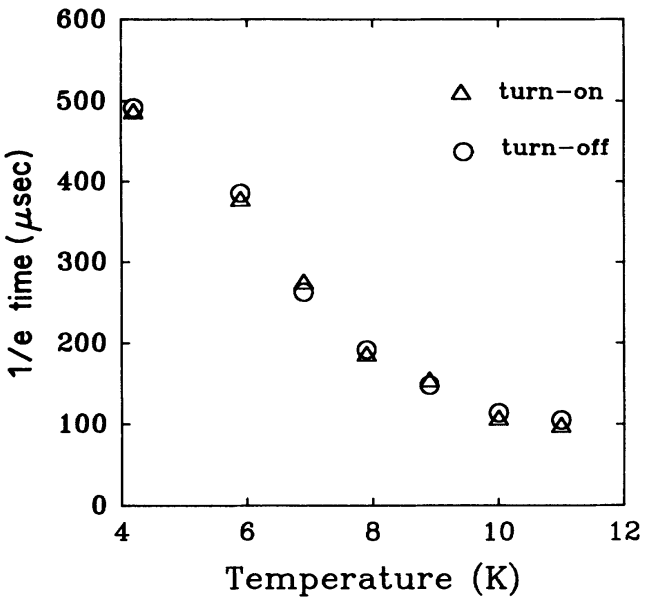

FIG. 7. 1/e time ( $\mu \mathrm{sec})$ for turn on and turn off of red emission, taken with proton-beam irradiation.

irradiated sample (to make sure the emission feature has reached its steady-state value) are shown in Fig. 6 for a variety of temperatures. One of the more interesting features of these timing curves is that the turn-on and turn-off curves are almost identical, even to the point where multiexponential fit parameters between the two show good agreement. The temperature dependence of the turn off suggest that the turn off is not due to any radiative lifetime, but instead the lifetime of some species in the solid.

The 1/e times (as defined in Ref. 4) for both turn-on and turn-off curves as a function of temperature are shown in Fig. 7. The large discrepancies between these lifetimes and those measured on the previously unirradiated sample suggest the presence of two different species, one of which turns on and off rapidly with irradiation. The lifetimes of the fast timing data show a region near 6 $\mathrm{K}$ where the lifetime decreases sharply. NMR studies of vacancy diffusion ${ }^{22}$ in solid $\mathrm{H}_{2}$ and $\mathrm{D}_{2}$ indicate that below $6 \mathrm{~K}$ all classical diffusion is frozen out, and the only transport mechanism is tunneling. The diffusion coefficient for vacancies in solid $D_{2}$ has also been calculated by Ebner and Sung ${ }^{23}$ and is shown to be extremely small below $6 \mathrm{~K}$ and rapidly increases for greater temperatures. The similarity between these temperatures and the lifetimes from the fast timing data suggests that the motion of the species involved in the red emission is at least to some extent a vacancy assisted process.

\section{DISCUSSION}

The red emission has the same spectrum as the optical flashes of Ref. 19, suggesting that they have the same origin. It is not obvious, however, that this origin has to be the unpaired atoms in the solid. In particular, a triggering thermal pulse would increase the concentration of many reactive species in the solid by "detrapping" them. If these species then reacted (radiatively) exothermally, the process would continue.

One might consider any number of processes involving a plethora of charged species in the sample. However, the behavior of the charged species has been studied for many years and all experiments have been explained by considering a large concentration of highly mobile electrons and positive ions (e.g., $\mathrm{D}_{3}{ }^{+}$) along with a smaller concentration of two far less mobile positive and negative charge species which are responsible for timing behavior on a scale of seconds to hours. ${ }^{24,4}$ More importantly, all of the immobile charge species anneal at temperatures of about $12 \mathrm{~K}$ and no previously measured phenomenon shows the distinction between a fresh and an annealed sample that Fig. 5 shows.

It is not so easy to exclude $\mathrm{D}_{3}$ emission as the cause of the continuum red emission since $\mathrm{D}_{3}{ }^{+}, e^{-}$recombination is occurring continuously and the known uv spectrum of $\mathrm{D}_{3}$ has been assigned to emission from solid deuterium. ${ }^{21,7}$ However, no 800 -nm feature is known or predicted for $\mathrm{D}_{3}$ (Ref. 25) and any dynamic modeling that we have tried cannot account for the behavior shown in Fig. 5.

Of all various species observed in hydrogen solids to date only nitrogen and oxygen atomic impurities ${ }^{21}$ have acted in the manner described by Fig. 5. These $\mathbf{N}$ and $\mathrm{O}$ impurities were not removed by annealing, and the relative number of impurity atoms to impurity molecules could only be restored by melting the sample. Since D atoms are comparable in size to oxygen and nitrogen counterparts it seems likely that D atoms are also unaffected by the annealing process. This, of course, is true only if the dominant mechanism for atom transport is physical diffusion rather than chemical diffusion. There has been no direct evidence, however, for atoms in any experiment capable of performing timing measurements, and so this conclusion is still tentative. The above arguments suggest that the origin of the red emission (and the optical flashes) is related to the disappearance of $\mathrm{D}$ atoms in the solid. The actual radiation process is still uncertain and we will only briefly mention some possible mechanisms.

A radiative transition from an excited electron bubble state could produce a broad emission continuum in the observed spectral region. ${ }^{7,24}$ Because the timing behavior of this red emission is so very different from that of the electron bubbles, one has to suppose that bubbles are formed rapidly in excited states and that the radiative decay is at least as rapid as the observed timing. However, the bubbles in their ground state are very much slower to turn on with the proton beam, which necessitates the existence of some long-lived intermediate state which is not predicted by our current models. ${ }^{24}$ Furthermore, some mechanism for transferring energy from atom-atom association into additional electron bubble formation would have to be proposed.

Radiative association of two ground-state $\mathrm{D}$ atoms does not occur following two-body collisions and is not expected to occur adiabatically. ${ }^{26}$ One can conceive of it occurring in the solid since atoms migrate by hopping between interstitial or substitutional sites. Two atoms could find themselves suddenly occupying the same substitutional site in which case "relaxing" into a molecular potential well would be probable. Nonradiative relaxa- 
tion is inhibited because $\hbar \omega$ between high vibrational levels of $\mathrm{D}_{2}$ is very much larger than the thermal $k T$ energies accessible to phonons. Radiation might then proceed either through the large admixture of molecular potentials available in the sudden approximation or through mediation of electron attachment to one of the atoms. Models based on the former mechanism are so far unable to produce the observed spectrum and the latter mechanism shows some promise in that the observed red emission spectrum looks very much like the photodetachment spectrum of $\mathrm{D}^{-}$which represents the inverse process. ${ }^{27}$ More experiments and further modeling will be required before an explanation of the red emission spectrum can be proffered.

\section{CONCLUSION}

We have presented a continuum emission spectrum from irradiated solid deuterium that has been studied in three different laboratories. The origin of the observed spectrum is uncertain, but is believed to be related to atomic association. Further experimental studies could try to monitor an unambiguous spectral signature of the atoms to examine this relation. Further theoretical work on atom-atom recombination in a matrix of solid deuterium would also be helpful.

\section{ACKNOWLEDGMENTS}

We would like to acknowledge financial support from the Natural Sciences and Engineering Research Council of Canada (NSERC) and the Danish Natural Science Research Council. Work performed under the auspices of the US Department of Energy by the Lawrence Livermore National Laboratory under Contract No. W-7405ENG-48. The LLNL group would also like to thank the NASA Lewis Research Center and USAF, Phillips Laboratory. Our thanks for technical support and help with the experiments are extended to J. Stark at McMaster Tandem Accelerator Laboratory, and D. Tokaryk and J. Miller at the University of Guelph.
*Permanent address: Hamburger Synchrotronstrahlungslabor (HASYLAB), Deutsches Electronen Synchrotron (DESY), D-2000 Hamburg, 52, Germany.

${ }^{1}$ P. C. Souers, E. M. Fearon, P. E. Roberts, R. T. Tsugawa, J. D. Poll, and J. L. Hunt, Phys. Lett. 77A, 277 (1980).

${ }^{2}$ R. L. Brooks, M. A. Selen, J. L. Hunt, Jack R. MacDonald, J. D. Poll, and J. C. Waddington, Phys. Rev. Lett. 51, 1077 (1983).

${ }^{3}$ M. A. Selen, R. L. Brooks, J. L. Hunt, J. D. Poll, Jack R. MacDonald, and J. C. Waddington, Nucl. Instrum. Methods B 230, 720 (1984).

${ }^{4}$ R. L. Brooks, S. K. Bose, J. L. Hunt, Jack R. MacDonald, and J. D. Poll, Phys. Rev. B 32, 2478 (1985).

${ }^{5}$ B. Stenum, J. Schou, H. Sørensen, and P. Gürtler, Radiat. Eff. Defects Solids 109, 235 (1989).

${ }^{6}$ J. A. Forrest, J. L. Hunt, and R. L. Brooks, Can. J. Phys. 68, 1247 (1990).

${ }^{7}$ J. Schou, B. Stenum, H. Sørensen, K.-V. Weisberg, and P. Gürtler, Nucl. Fusion 31, 589 (1991).

${ }^{8}$ S. K. Bose and J. D. Poll, Can. J. Phys. 63, 94 (1985).

${ }^{9}$ S. K. Bose and J. D. Poll, Can. J. Phys. 63, 1105 (1985).

${ }^{10}$ S. K. Bose and J. D. Poll, Can. J. Phys. 65, 67 (1987).

${ }^{11}$ C. K. Jen, S. N. Foner, E. L. Cochran, and V. A. Bouers, Phys. Rev. 112, 1169 (1956).

${ }^{12}$ L. H. Piette, R. C. Rempel, H. E. Weaver, and J. M. Flournay,
J. Chem. Phys. 30, 1623 (1959).

${ }^{13}$ Harvey N. Rexroad and Walter Gordy, Phys. Rev. 125, 242 (1962).

${ }^{14}$ R. W. H. Webeler, J. Chem. Phys. 64, 2253 (1976).

${ }^{15}$ Gerald Rosen, J. Chem. Phys. 66, 5423 (1977).

${ }^{16}$ Gerald Rosen, J. Chem. Phys. 65, 1735 (1976).

${ }^{17}$ Frank J. Zeleznik, J. Chem. Phys. 65, 4492 (1976).

${ }^{18}$ G. W. Collins, E. M. Fearon, J. L. Maienschein, E. R. Mapoles, R. T. Tsugawa, and P. C. Souers, Phys. Rev. Lett. 65, 444 (1990).

${ }^{19}$ E. R. Mapoles, F. Magnotta, G. W. Collins, and P. C. Souers, Phys. Rev. B 41, 11653 (1990).

${ }^{20}$ Robert L. Brooks, J. Chem. Phys. 85, 1247 (1986).

${ }^{21}$ Bjarne Stenum, Ph.D. thesis, Ris $\varnothing$ National Laboratory, 1991.

${ }^{22}$ D. Zhou, M. Rall, J. P. Brison, and N. S. Sullivan, Phys. Rev. B 42, 1929 (1990).

${ }^{23}$ C. Ebner and C. C. Sung, Phys. Rev. A 5, 2625 (1972).

${ }^{24}$ R. L. Brooks and J. L. Hunt, Phys. Can. 47, 132 (1991), and references therein.

${ }^{25}$ I. D. Petsalakis, G. Theodorakopoulos, and J. S. Wright, J. Chem. Phys. 89, 6850 (1988).

${ }^{26}$ William B. Latter and John H. Black, Astro. J. 372, 161 (1991).

${ }^{27}$ Stephen J. Smith and David S. Burch, Phys. Rev. 116, 1125 (1959). 